\title{
VIABILITY IN DROSOPHILA MELANOGASTER IN RELATION TO AGE AND ADH ACTIVITY OF EGGS TRANSFERRED TO ETHANOL FOOD
}

\author{
ELS BIJLSMA-MEELES \\ Biology Centre, Department of Genetics, University of Groningen, \\ Haren -Gn*, The Netherlands
}

Received 12.viii.78

\begin{abstract}
Summary
Egg-to-adult survival of Adh genotypes of Drosophila melanogaster on ethanol food appears to depend on the age at which the eggs are transferred from regular to ethanol-containing food. Young eggs give much higher viability than old eggs. This seems to be due to an increased ethanol tolerance in eggs exposed to ethanol medium. Additionally it is shown that ADH activity in ethanol-treated eggs has increased as compared with non-treated eggs. It is argued, however, that it is not this increase in ADH activity but other, still unknown, factors which are responsible for ethanol tolerance in eggs and larvae. The relation between ADH activity and fitness of $A d h$ genotypes in environments containing alcohols is discussed.
\end{abstract}

\section{INTRODUCTION}

AN important problem for population geneticists is finding what proportion of the allozyme variation found in natural populations of many species is associated with fitness differences, and what proportion is effectively neutral? (see e.g. Kimura and Ohta, 1971; Lewontin, 1974, for extensive treatments of the selectionist-neutralist controversy).

The obvious way to get an unambiguous answer to this question is to determine the relative fitnesses of the different genotypes, preferably in a variety of environments. By choosing environments relevant to the properties of the enzyme under study, the chance of detecting selective differences due to the locus itself is greatly increased. Such relationships between the properties of allozymes and the survival of corresponding genotypes in relevant environments have been found, for instance, in the case of the amylase locus (De Jong and Scharloo, 1976) and the alcohol dehydrogenase locus of Drosophila melanogaster. With respect to the latter locus, several reports contain evidence that selection is operating. Selection was found to favour the $\mathrm{F}$ allele (notation according to Grell et al., 1965) when ethanol (Gibson, 1970; Bijlsma-Meeles and Van Delden, 1974) or one of several other alcohols (Van Delden et al., 1975) was added to the food. Morgan (1975), in competition experiments with larvae on food with different alcohols, found in most cases an advantage of the FF genotype over the SS genotype. However, when adult flies were exposed to the vapour of 1penten-3-ol, an alcohol known to give a toxic product of oxidation, more flies of the FF genotype died. From in vitro measurements using ethanol and several other alcohols as substrates (Gibson, 1970; Vigue and Johnson, 1973; Day et al., 1974a, b) it appears that FF homozygotes possess higher 
$\mathrm{ADH}$ activity than SS homozygotes, whereas heterozygotes have intermediate activity. Thompson and Kaiser (1977) demonstrated a positive correlation between the ADH activity of some strains and their egg-to-adult survival on alcohol supplemented media.

These results strongly support the idea that survival on media containing alcohols is directly related to $\mathrm{ADH}$ activity. However, this relationship does not appear to be a straightforward one in other reports. Oakeshott (1976) found the survival of heterozygous adults on ethanol food to be higher than that of both homozygous genotypes, whereas the $\mathrm{ADH}$ activity of the former was intermediate. However, the pre-adult developmental times of the different $A d h$ genotypes did show a relationship with $\mathrm{ADH}$ activity. Briscoe et al. (1975) found no difference in ethanol tolerance between adults of the FF and FS genotypes. McKenzie and Parsons (1974) found no difference in the frequency of the $\mathrm{F}$ allele in a population of Drosophila melanogaster within and outside a wine cellar, whereas the ethanol tolerance of flies in and outside the cellar was found to be different. Ward (1974, 1975) and Hewitt et al. (1974) have shown that considerable genetic variation in $\mathrm{ADH}$ activity exists both among homozygous FF flies and among homozygous SS flies. If $\mathrm{ADH}$ activity is an important factor in determining survival on alcohol-containing media, one expects selection for higher activity levels to occur in populations kept for many generations on ethanol food. This has not yet been demonstrated (Van Dijk, unpublished).

It appears then that the relation between $\mathrm{ADH}$ activity and survival in ethanol-containing environments has not been completely elucidated. The results of the experiments presented here show that the survival of the various Adh genotypes of Drosophila melanogaster on ethanol food does not simply depend on $\mathrm{ADH}$ activity as measured in vitro, but is greatly influenced by other factors. The experiments were started to discover the reason why there was a difference in survival on ethanol food between experiments carried out on various occasions in spite of equal ethanol concentration in the food (Bijlsma-Meeles, unpublished results). The question asked was whether the age of eggs of the three $A d h$ genotypes, at the moment of transfer had any influence on subsequent egg-to-adult survival. In addition the $\mathrm{ADH}$ activity of eggs from the three genotypes was assayed both after exposure to normal medium and after exposure to a medium supplemented with ethanol.

\section{Materials AND METHOdS}

\section{(i) Strains}

From the Groningen population (Bijlsma-Meeles and Van Delden, 1974) 27 homozygous fast (F) lines, and 27 homozygous slow (S) lines (notation according to Grell et al., 1965) were obtained from single-pair crosses between identical homozygous parents. All the lines that showed the same genotype were mixed and distributed over 10 bottles. Thereafter they were maintained at $25^{\circ} \mathrm{C}$ and 70 per cent $\mathrm{RH}$ on regular food medium as one population by mixing the flies from all the bottles every 14 days when transferring them to fresh bottles. Ten months after the foundation of the homozygous populations, flies from them were used in the experiments to be reported. 


\section{(ii) Food media}

Normal medium contained $19 \mathrm{~g}$ agar, $54 \mathrm{~g}$ sucrose, $32 \mathrm{~g}$ yeast, $13 \mathrm{ml}$ nipagin solution ( $10 \mathrm{~g}$ nipagin in $100 \mathrm{ml}$ ethanol 96 per cent) in $1 \mathrm{l}$ water. Ethanol medium was obtained by adding $12 \mathrm{ml}$ ethanol (96 per cent) to $88 \mathrm{ml}$ normal medium with vigorous stirring, after the latter had been cooled to $50^{\circ} \mathrm{C}$. Ethanol food was stored at $4^{\circ} \mathrm{C}$ and used about 24 hours after preparation.

\section{(iii) Egg collecting}

Virgin flies, grown at $25^{\circ} \mathrm{C}$ and 70 per cent relative humidity on normal medium in uncrowded conditions were collected and crossed in order to obtain eggs of the required genotypes. To stimulate egg production flies were fed with additional live yeast during 3 days, before transferring them to the egg laying vials. These vials contained a thin $(5 \mathrm{~mm})$ layer of agar ( 2 per cent solution in tap water) for egg laying and live yeast for feeding. To obtain egg laying medium containing 12 per cent ethanol, the agar solution was cooled to $50^{\circ} \mathrm{C}$ and the appropriate amount of ethanol was added with stirring. Egg laying media were always used within a few hours after preparation. After a few hours of egg laying the flies were discarded. For egg collecting the eggs were washed from the agar surface with tap water.

\section{(iv) Age experiment}

To investigate the influence of age of transfer to ethanol food on subsequent egg-to-adult survival, an experiment was performed as follows. Eggs laid on egg-laying medium without ethanol by 10 to 12-day-old flies during a period of 4 hours were kept on this medium for various periods before they were transferred to vials containing $9 \mathrm{ml}$ ethanol food. Eggs transferred immediately to the ethanol food after the egg-laying period of 4 hours are referred to as eggs with a mean age of 2 hours. Eleven tests have been done, five of which were started with eggs (mean age 2, 6, 10, 14 and 18 hours respectively), one with about equal numbers of eggs and larvae (22 hours), and four with larvae only (26, 30, 63 and 78 hours). In addition a test was carried out in which eggs were laid on egg-laying medium containing 12 per cent ethanol and thereafter (at a mean age of 18 hours) transferred to the vials containing ethanol food. Each test was started with 12 vials of each of the Adh genotypes: SS, FF, SF and FS (the first letter refers to the genotype of the mother). Each vial was started with 50 individuals (eggs or larvae). From each vial the hatched adults were collected daily and counted.

\section{(v) ADH activity assay}

In order to determine whether ADH activity in eggs changes after exposure to ethanol the following experiment has been done. Eggs were laid by 6 to 8-day-old flies during 3 hours on egg-laying medium with or without 12 per cent ethanol, and kept on these media until a sample was taken for ADH activity assay, the age of the FF, SS and FS eggs at that moment being 16, 17 and 18 hours respectively. Egg samples of a given genotype but originating from different media were assayed alternately. 
Four replicate samples of each genotype were used from both media. Each sample consisted of an amount of eggs of known weight. About $5 \mathrm{mg}$ appeared to be suitable for the assay, which was carried out according to the method of Van Delden et al. (1975). In order to calculate the enzyme activities of individual eggs the weight of eggs originating from normal and ethanol medium was determined. Five replicate samples (of 50 eggs) of each genotype and treatment were weighed for this purpose.

\section{Results}

\section{(i) Age experiment}

Fig. 1 shows survival of eggs and larvae of the different age groups on food medium supplemented with ethanol. The time of egg transfer from normal to ethanol medium appears to have great influence on survival of all genotypes. Young larvae raised on medium without ethanol all died

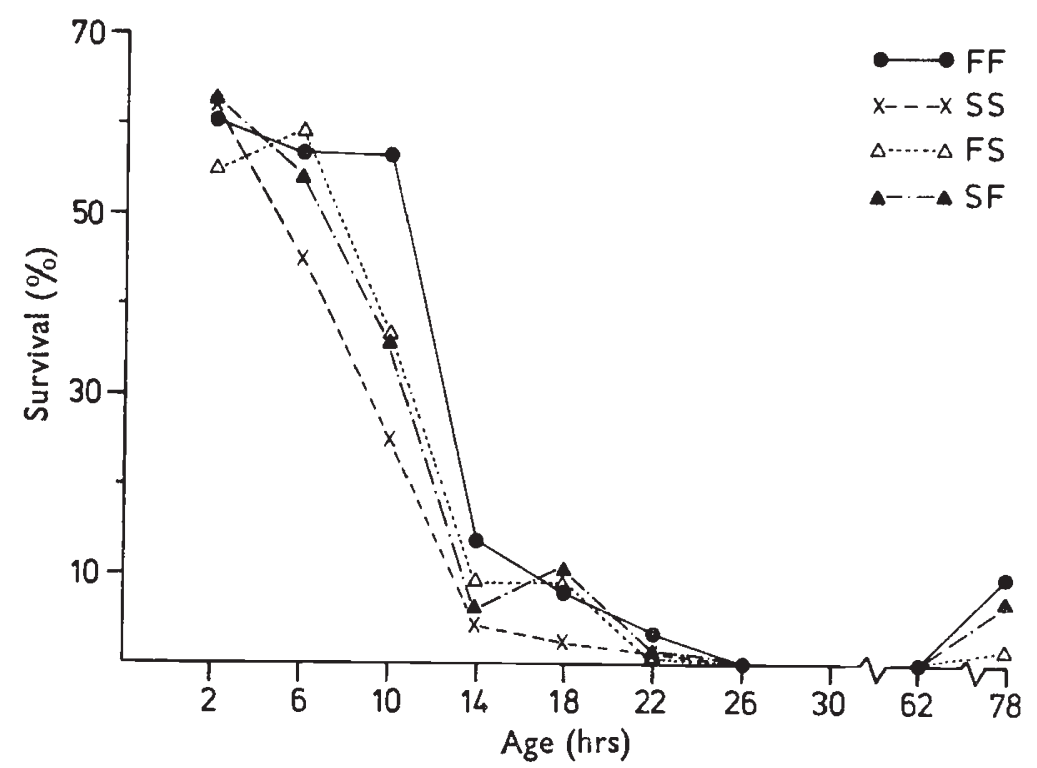

FIG. 1.-Percentage survival into adults from eggs or larvae, transferred to food supplemented with 12 per cent ethanol at different ages.

when transferred to ethanol food. If eggs were transferred to this food, however, a variable fraction of adults developed. From eggs transferred at an early stage to the ethanol food and consequently exposed to it for a longer period before hatching, more adults developed than from older eggs. Also, when third instar larvae were transferred to ethanol food, some adults hatched (except in the case of the SS genotype). The survivors in this case most probably developed from larvae ready to pupate when transferred. It was observed that these larvae had a tendency to avoid the food and immediately to climb the walls of the vials. As well as the differences in 
survival between age groups there are differences between genotypes within age groups. These differences were tested for their significance by means of an analysis of variance on survival percentages (expressed as angles) for each age group separately, with an additional Tukey's t-test for multiple comparisons. The figures of table 1 show that in almost all age groups there are significant differences: SS genotypes always showing less survival than FF genotypes. Only from eggs with a mean age of two hours did equal percentages of the different genotypes hatch. Special attention is drawn to the experiment where the eggs were laid on ethanol medium instead of on medium without ethanol before transferring them to the vials with ethanol food (table 1, age group 18*). The percentage of hatched adults in this case was lower than in the experiment started with eggs of 2 hours,

\section{TABLE 1}

Percentage hatched adults (means of 12 replicates) from eggs or larvae transferred at different ages to food supplemented with 12 per cent ethanol. Genotypes not listed on a common line, differed significantly from each other at the 0.05 level (Tukey's $t$-test for multiple comparisons)

Developmental stage

Eggs

Eggs

About equal numbers eggs and larvae
Mean age (hours) Percentage hatched adults

\begin{tabular}{|c|c|c|c|c|}
\hline \multirow[t]{2}{*}{2} & SF & SS & FF & FS \\
\hline & $60 \cdot 2$ & $61 \cdot 7$ & 60.5 & $54 \cdot 8$ \\
\hline \multirow[t]{2}{*}{6} & FS & FF & SF & SS \\
\hline & $59 \cdot 3$ & $56 \cdot 7$ & $54 \cdot 3$ & $45 \cdot 0$ \\
\hline \multirow[t]{2}{*}{10} & FF & $\underline{\text { FS }}$ & SF & SS \\
\hline & $56 \cdot 7$ & $36 \cdot 7$ & $36 \cdot 0$ & $25 \cdot 0$ \\
\hline \multirow[t]{2}{*}{14} & FF & FS & SF & SS \\
\hline & $13 \cdot 5$ & $9 \cdot 3$ & $6 \cdot 0$ & 3.7 \\
\hline \multirow[t]{2}{*}{18} & SF & $\underline{\text { FS }}$ & FF & SS \\
\hline & $10 \cdot 0$ & $9 \cdot 0$ & $8 \cdot 0$ & $2 \cdot 5$ \\
\hline \multirow[t]{2}{*}{$18 *$} & SF & FF & $\underline{\text { FS }}$ & SS \\
\hline & 53.5 & $51 \cdot 5$ & $44 \cdot 7$ & 43.5 \\
\hline \multirow[t]{2}{*}{22} & FF & SF & SS & FS \\
\hline & $3 \cdot 2$ & 1.3 & 0.8 & 0.2 \\
\hline
\end{tabular}

Larvae $\quad\left\{\begin{array}{cccccc}26 & \multicolumn{4}{c}{\text { No survival }} \\ 30 & \multicolumn{4}{c}{\text { No survival }} \\ 62 & \multicolumn{4}{c}{\text { No survival }} \\ 78 & \frac{\text { FF }}{9.8} & \frac{\text { SF }}{7 \cdot 3} & \frac{\text { FS }}{\text { SS }} & 1 \cdot 3 & 0\end{array}\right.$

* Eggs laid on ethanol medium instead of on medium without ethanol and transferred to the experimental vials at the age of 18 hours. 
but was much higher than in the other experiment started with eggs of 18 hours, which were laid on egg-laying medium without ethanol. A separate control experiment was performed to investigate the possibility that the differences in viability connected with the transfer from egg-laying medium to ethanol medium, described in the previous section, were merely caused by the lack of sucrose and nipagin in the former medium. In this experiment homozygous SS eggs, laid on this egg-laying medium or on regular food medium during a period of 4 hours, were transferred both to normal and to ethanol medium, either immediately or after 14 hours. In this way eight tests, each consisting of 10 replicate vials (each with 50 eggs), were carried out under conditions identical to those in the previous experiments. The results are given in table 3A. From the analysis of variance (table 3B), performed after angular transformation of the data in table $3 \mathrm{~A}$ it appeared that the medium on which the eggs were laid had no effect on viability. Comparable to the previous experiments however, it was found that both the age of the eggs at transfer and the medium to which the eggs were transferred affected viability significantly.

From these results it appears that under the influence of environmental ethanol a tolerance against ethanol can develop in eggs. The responsible mechanism could possibly be an increase in $\mathrm{ADH}$ activity.

\section{(ii) $A D H$ activity assay}

The results of this experiment are listed in table 2. Means and standard errors of $\mathrm{ADH}$ activity per mg egg weight and weight per 50 eggs are given in the first two rows, both for ethanol treated and control eggs of the three Adh genotypes. It can easily be seen that after exposure to ethanol, eggs of all genotypes have higher $\mathrm{ADH}$ activity/mg than control eggs, whereas their weight is lower than of the control eggs. All these differences, tested

TABLE 2

Means and standard errors (S.E.) of ADH activity and weight of eggs of three Adh genotypes after exposure to a 12 per cent ethanol medium (e) and control medium (c). Differences are tested for significance by means of a student's $t$-test for each genotype separately and the probability $P$ is given. ADH activity is expressed as $\Delta E .10^{3} / \mathrm{min}$

\begin{tabular}{|c|c|c|c|c|c|c|}
\hline \multirow{3}{*}{$\begin{array}{l}\text { ADH activity } / \mathrm{mg} \\
\text { S.E. }\end{array}$} & \multicolumn{2}{|c|}{$\mathrm{FF}$} & \multicolumn{2}{|c|}{ SS } & \multicolumn{2}{|c|}{$\mathrm{FS} / \mathrm{SF}^{*}$} \\
\hline & $\begin{array}{c}c \\
3 \cdot 94 \\
(0 \cdot 12)\end{array}$ & $\begin{array}{c}e \\
4 \cdot 72 \\
(0 \cdot 22)\end{array}$ & $\begin{array}{c}c \\
1 \cdot 60 \\
(0 \cdot 04)\end{array}$ & $\begin{array}{c}e \\
2 \cdot 17 \\
(0 \cdot 17)\end{array}$ & $\begin{array}{c}c \\
2 \cdot 89 \\
(0 \cdot 09)\end{array}$ & $\begin{array}{c}e \\
4 \cdot 26 \\
(0 \cdot 14)\end{array}$ \\
\hline & \multicolumn{2}{|c|}{$0.01<\mathrm{P}<0.05$} & \multicolumn{2}{|c|}{$0.01<\mathrm{P}<0.05$} & \multicolumn{2}{|c|}{$\mathrm{P}<0.001$} \\
\hline \multirow[t]{2}{*}{$\begin{array}{l}\text { Weight of } 50 \text { eggs (mg) } \\
\text { S.E. }\end{array}$} & $\begin{array}{c}0.525 \\
(0.006)\end{array}$ & $\begin{array}{c}0.489 \\
(0.003)\end{array}$ & $\begin{array}{c}0.541 \\
(0 \cdot 006)\end{array}$ & $\begin{array}{c}0.510 \\
(0.003)\end{array}$ & $\begin{array}{c}0.528 \\
(0.004)\end{array}$ & $\begin{array}{c}0.504 \\
(0 \cdot 002)\end{array}$ \\
\hline & \multicolumn{2}{|c|}{$0.005<\mathrm{P}<0.01$} & \multicolumn{2}{|c|}{$0.001<\mathrm{P}<0.005$} & \multicolumn{2}{|c|}{$P<0.001$} \\
\hline \multirow[t]{2}{*}{$\begin{array}{l}\text { ADH activity } / \mathrm{egg} \times 10^{2} \\
\text { S.E. }\end{array}$} & $\begin{array}{c}4 \cdot 14 \\
(0 \cdot 13)\end{array}$ & $\begin{array}{c}4 \cdot 70 \\
(0 \cdot 22)\end{array}$ & $\begin{array}{c}1 \cdot 73 \\
(0 \cdot 04)\end{array}$ & $\begin{array}{c}2 \cdot 22 \\
(0 \cdot 18)\end{array}$ & $\begin{array}{c}3 \cdot 06 \\
(0 \cdot 10)\end{array}$ & $\begin{array}{c}4 \cdot 31 \\
(0 \cdot 14)\end{array}$ \\
\hline & \multicolumn{2}{|c|}{$0.05<\mathrm{P}<0.1$} & \multicolumn{2}{|c|}{$0.01<P<0.05$} & \multicolumn{2}{|c|}{$P<0.001$} \\
\hline
\end{tabular}

* Heterozygous eggs used for ADH activity assay were of the FS genotype. For determination of weight a mixture of equal numbers of eggs of the FS and SF genotype was used. 
by means of a Student's t-test are significant. The increase in $\mathrm{ADH}$ activity after treatment with ethanol, expressed per mg egg weight, could be caused by a lower weight of these eggs, as samples of equal weight contain more ethanol-treated than control eggs. For this reason $\mathrm{ADH}$ activities per egg were also calculated, by correcting the values of each of the four replicate measurements of $\mathrm{ADH}$ activity/mg for the values determined for the weight of 50 eggs. In this way four replicate values of activity/egg were obtained for each genotype; their means and standard errors are given in the third row of table 2. ADH activity expressed per individual egg appears to be higher after the ethanol treatment, the difference being significant except for the FF genotype.

\section{Discussion}

The question whether differences in age of eggs at the moment of transfer to ethanol food have any influence on egg-to-adult survival can be answered positively. The results show clearly a negative correlation between the age of eggs and the percentage of hatching adults on food containing 12 per cent ethanol. Transferring young larvae to this food can lead to 100 per cent mortality. As a difference of only a few hours in the age of the eggs already causes substantial differences in survival (especially around an age of 12 hours) it will be apparent that this effect can have important consequences for the outcome of experimental work.

The possibility that the phenomenon is due to the experimental design needs to be considered. The ethanol concentrations in the food, though equal at the start of each test, when the eggs are transferred, will gradually decrease by evaporation during the experimental period. In tests started with relatively old eggs, larvae will hatch sooner after the start of the experiment than in tests started with younger eggs. Consequently the former larvae may have to cope with a higher ethanol concentration in the food, which in turn, may result in a higher mortality of these larvae. Considering the results of two tests started with eggs of equal age, but laid previously on different media (table 1: age groups of 18 hours), it appears that this cannot be the whole explanation. At least 30 per cent more flies hatched from the eggs that had been laid on ethanol medium than from eggs originating from medium without ethanol. From this it appears that previous exposure of eggs to ethanol medium increases subsequent survival on ethanol food. And though in a preliminary experiment it was found that eggs on a 12 per cent ethanol medium hatch a few hours later than on contol medium, it is difficult to conceive that a decrease in ethanol concentration in such a short period is sufficient to cause such a difference in survival. That the decline in viability is not caused by the initial stay on egg-laying medium, lacking sucrose and nipagin, is clearly demonstrated by table 3 . Again it is shown that young eggs transferred to ethanol medium show a much higher survival than older eggs, though the survival is lower than in the first experiment (table 1). As the control experiment was performed more than 1 year after the original experiment, it is likely that changes in viability had occurred in the strains leading to an overall decline in viability.

As there seem to be sufficient arguments to exclude the possibility that the age effect is an artefact of the particular methods used, the most likely explanation is that exposure of eggs in an early developmental phase or 
during a certain length of time leads to increased ethanol tolerance in eggs or larvae.

It is further shown that eggs have higher ADH activity after exposure to ethanol, though the difference was not significant in the case of eggs of the FF genotype, when activity was expressed per egg. Substrate induced changes in $\mathrm{ADH}$ activity have been reported by Horikawa et al. (1967) and Gibson (1970) for embryonic cells and larvae of Drosophila melanogaster respectively. To observe them also in Drosophila eggs that must be rather impermeable to ethanol, as they resist temporal submersion in 75 per cent ethanol without much harm (Lazarus et al., 1960), is unexpected. However,

TABLE 3

Control experiment. A. Percentages egg-to-adult survival of SS-eggs laid on egg-laying medium (agar) and regular food medium and transferred to regular and ethanol-food medium at an age of 2 or 16 hours. Each entry is based on 10 replicate vials (each with 50 eggs). B. Analysis of variance of the data in $A$ (after angular transformation)

A. Eggs laid on

Agar medium

Regular medium

Agar medium

Regular medium

B. Source

Egg laying medium $(E)$

Food medium $(F)$

Age of eggs $(A)$

$E \times F$

$F \times A$

$E \times A$

$E \times F \times A$

Error

\begin{tabular}{ccc} 
Age of eggs & \multicolumn{2}{c}{ Percentage survival } \\
& Regular food & Ethanol food \\
at transfer $(h)$ & $74 \cdot 6$ & $27 \cdot 0$ \\
2 & $80 \cdot 2$ & $29 \cdot 4$ \\
16 & $80 \cdot 4$ & 0 \\
16 & $75 \cdot 4$ & $2 \cdot 0$
\end{tabular}

$\begin{array}{ccc}\text { d.f. } & \text { Mean square } & \text { F-value } \\ 1 & 0 \cdot 44 & <1 \\ 1 & 40844.53 & 288 \cdot 80^{*} \\ 1 & 4464 \cdot 67 & 31 \cdot 57 * \\ 1 & 0 \cdot 64 & <1 \\ 1 & 4702 \cdot 32 & 33 \cdot 25 * \\ 1 & 134.58 & <1 \\ 1 & 19 \cdot 90 & <1 \\ 72 & 141 \cdot 43 & \\ * \mathrm{P}<0.001 & & \end{array}$

very small amounts of ethanol may be sufficient to produce these changes. Small amounts could pass, either from solution or as vapour, through the micropyle that is believed to penetrate the vitelline membrane (King and Koch, 1963) even if the membrane itself was impermeable. It remains possible, however, that the observed changes in the egg are secondary effects of the ethanol treatment, connected for instance with dehydration or changes in the covering membranes.

The experiment described here was designed to compare ethanoltreated and control eggs within, rather than between, genotypes, as eggs of the various genotypes were exposed to ethanol for slightly different periods. For this reason the results cannot be compared with Gibson's finding that larvae of the various $A d h$ genotypes showed a differential increase in $\mathrm{ADH}$ activity. The relative differences between genotypes in $\mathrm{ADH}$ activity found in control eggs are significant and comparable to the differences found generally in larvae and adults: FF $>$ FS $>$ SS. Also the activity in ethanol-treated FF eggs is significantly higher than in ethanoltreated SS eggs. 
Considering each genotype separately it seems likely that the increased ethanol tolerance developed in eggs under influence of environmental ethanol is due to the higher ADH activity at the same time induced in these eggs. Comparison of the data for the various genotypes, on the contrary, leads to the conclusion that there can be no causal relationship between survival and $\mathrm{ADH}$ activity, at least not absolute $\mathrm{ADH}$ activity as measured in vitro. It is true that, after exposure of SS eggs to ethanol, $\mathrm{ADH}$ activity and egg-to-adult survival are considerably increased, but the enzyme activity is still lower than that of unexposed FF eggs, which survive very poorly.

Apparently factors other than absolute $\mathrm{ADH}$ activity underlie the ethanol tolerance of Drosophila melanogaster. This is in accordance with the finding of McKenzie and Parsons (1974) of a difference in ethanol tolerance between adult flies in and outside a wine cellar, whereas there was no difference in genotypic composition with respect to the Adh locus. The development of a tolerance to ethanol distinct from the $\mathrm{ADH}$ system would also account for the lack of selection for high $\mathrm{ADH}$ activity in populations kept on ethanol food (see Introduction). The rise in $\mathrm{ADH}$ activity influenced by ethanol is apparently of little adaptive significance. It could be part of a response by the organism to stress (in this case high ethanol concentration). It might be interesting to investigate other enzymes in this respect as well. The nature of the induced ethanol tolerance is still obscure but the fact that the permeability of membranes can change under the influence of ethanol may be relevant (Chin and Goldstein, 1977).

So far the results seem to demonstrate that $\mathrm{ADH}$ activity and survival on ethanol food have no causal relationship. But how do we explain the fact that the differences of activity in vitro found between $A d h$ genotypes (Rasmuson et al., 1966; Gibson, 1970; Vigue and Johnson, 1973, Day et al., 1974a, b) show a suggestive consistency with fitness differences demonstrated in the presence of alcohols (Gibson, 1970; Bijlsma-Meeles and Van Delden, 1974; Morgan, 1975; Thompson and Kaiser, 1977)? Also, in the age experiment (table 1) FF genotypes have significant higher survival than SS genotypes on ethanol food in most age groups. Given the suggestion that $\mathrm{ADH}$ activity is not important for survival, it seems inappropriate to ascribe the better survival of the FF genotype to the higher in vitro $\mathrm{ADH}$ activity. Perhaps there is a possibility that differences other than enzyme activity between the Adh genotypes are playing a role. The fact that selection also favours the $\mathrm{F}$ allele on food with methanol, which is not metabolised by ADH (Van Delden et al., 1975) seems to support this.

There is another interesting aspect in the data of table 1. Survival of the SS genotype, when expressed as a percentage of that of the FF genotype, rises from about 25 per cent in the relatively old age groups to about 90 per cent in the younger age groups. The difference even vanishes when the eggs are only 2 hours old. If the latter fact is not due to chance this could mean that the differences are not found unless the organism is exposed to rather abrupt changes in ethanol concentration. This certainly needs further investigation as in the majority of the cases where fitness differences between Adh genotypes are demonstrated on ethanol medium, eggs, larvae or adults are transferred to this food after they had been laid or grown on media without ethanol. New experiments will be needed to determine if the differences still exist when the whole life cycle is accomplished on ethanol food. No 
conclusion can be drawn from the one test in which the eggs were laid on ethanol medium (table $1,18^{*}$ hours) since it is likely that due to evaporation the ethanol concentration in the thin layer of egg-laying medium has become lower than on the ethanol food to which the eggs were transferred after 18 hours. It is important to do such experiments, because it is unlikely that flies in natural populations are subject to such abrupt changes in ethanol concentration. It is also evident that considering the relation between $\mathrm{ADH}$ activity and survival on ethanol media one has to be aware that the activity of a particular genotype cannot be regarded as a constant. It varies within genotypic classes (Ward, 1974), in the course of development (Dunn et al., 1969; Wright and Shaw, 1970; Hewitt et al., 1974) and under the influence of environmental ethanol, as shown in this paper and by Gibson (1970) and Horikawa et al. (1967).

Acknowledgments.-I would like to thank A. Kamping for assistance with the experimental work and Drs R. Bijlsma, Dr W. van Delden and Professor W. J. Feenstra for critically reading the manuscript. Thanks are due to Mrs L. Krol and Mrs P. Scheperkamp for typing the manuscript.

\section{REFERENGES}

BIJLSMA-MEELES, E., AND VAN DELDEN, w. 1974. Intra- and inter-population selection concerning the alcohol dehydrogenase locus in Drosophila melanogaster. Nature, 247, 369-371.

BRISCOE, D. A., ROBERTSON, A., AND MALPICA, J. M. 1975. Dominance at $A d h$ locus in response of adult Drosophila melanogaster to environmental alcohol. Nature, 255, 148-149.

GHIN, J. H., AND GOLDSTEIN, D. B. 1977. Drug tolerance in biomembranes: a spin label study of the effects of ethanol. Science, 196, 684-685.

DAY, T. H., HILlIER, P. C., AND CLARKE, B. 1974a. Properties of genetically polymorphic isozymes of alcohol dehydrogenase in Drosophila melanogaster. Biochem. Genet., 11, 141-153.

DAY, T, H., HILLIER, P. C., AND CLARKE, B. 1974b. The relative quantities and catalytic activities of enzymes produced by alleles at the alcohol dehydrogenase locus in Drosophila melanogaster. Biochem. Genet., 11, 155-165.

van DELDEN, w., KAMPING, A., AND VAN DIJK, H. 1975. Selection at the alcohol dehydrogenase locus in Drosophila melanogaster. Experientia, 31, 418-419.

DUNN, G. R., WILsON, T. G., AND JACOBSON, K. B. 1969. Age-dependent changes in alcohol dehydrogenase in Drosophila., F. Exp. Zool., 171, 185-190.

GiBson, J. 1970. Enzyme flexibility in Drosophila melanogaster. Nature, 227, 959-960.

GRELL, E. H., JACOBSON, к. B., AND MURPHY, J. B. 1965. Alcohol dehydrogenase in Drosophila melanogaster: isozymes and genetic variants. Science, $149,80-82$.

heWITT, N. E., PIPKIN, S. B., Williams, N., AND chakrabarTTY, P. K. 1974. Variation in $\mathrm{ADH}$ activity in class I and class II strains of Drosophila. F. Hered., 65, 141-148.

HORIKAWA, M., LING, L. N. L., AND FOX, A. s. 1967. Effects of substrates on gene-controlled enzyme activities in cultured embryonic cells of Drosophila. Genetics, 55, 569-583.

DE JONG, G., AND SCHARLOO, w. 1976. Environmental determination of selective significance or neutrality of amylase variants in Drosophila melanogaster. Genetics, 84, 77-94.

kimura, M., AND OHTA, T. 1971. Theoretical Aspects of Population Genetics. Princeton University Press, Princeton, N.J.

KING, R. C., AND KOCH, E. A. 1963. Studies on the ovarian follicle cells of Drosophila. Quart. F. micr. Sci., 104, 297-320.

LAZARUS, H. D., KELLY, G. L., AND SEDEROFF, R. 1960. Improved laying dishes and a refined technique for collecting and washing eggs. Drosophila Information Service, 34, 115.

Lewontin, R. C. 1974. The Genetics of Evolutionary Change. Columbia University Press.

MCKENZIE, J. A., AND PARSONS, P. A. 1974. Microdifferentiation in a natural population of Drosophila mleanogaster to alcohol in the environment. Genetics, 77, 385-394.

MORGAN, P. 1975. Selection acting directly on an enzyme polymorphism. Heredity, 34, $124-127$.

OAKESHOTT, J. G. 1976. Selection at the alcohol dehydrogenase locus in Drosophila melanogaster imposed by environmental ethanol. Genet. Res., 26, 265-274. 
RASMUSON, B., NILSON, L. R., AND RASMUSON, M. 1966. Effects of heterozygosity on alcohol dehydrogenase (ADH) activity in Drosophila melanogaster. Hereditas, 56, 313-316.

THOMPSON, J. N. JR., AND KAISER, T. N. 1977. Selection acting upon slow-migrating Adh alleles differing in enzyme activity. Heredity, 38, 191-195.

vIGUE, C. L., AND JOHNSON, F. M. 1973. Isozyme variability in species of the genus Drosophila. VI. Frequency-property-environment relationships of allelic alcohol dehydrogenases in D. melanogaster. Biochem. Genet., 9, 213-227.

WARD, R. D. 1974. Alcohol dehydrogenase in Drosophila melanogaster: activity variation in natural populations. Biochem. Genet., 12, 449-458.

WARD, R. D. 1975. Alcohol dehydrogenase activity in Drosophila melanogaster: a quantitative character. Genet. Res., 26, 81-93.

WRIGHT, D. A., AND SHAW, C. R. 1970. Time of expression of genes controlling specific enzymes in Drosophila embryos. Biochem. Genet., 4, 385-394. 